\section{Syncope and sudden death from the emergency physician's perspective: is there room for new biomarkers?}

\author{
Rossella Marino, ${ }^{1}$ Federico Floridi, ${ }^{2}$ \\ Clifford Mann, ${ }^{3}$ Andrea Semplicini, ${ }^{4}$ \\ Cinzia Sighieri, ${ }^{5}$ Enrico Lupia, ${ }^{6}$ \\ Pietro Lentini, ${ }^{7}$ Carlo Racani, ${ }^{8}$ \\ Salvatore Di Somma ${ }^{9}$ \\ 1 Medical-Surgery Sciences and \\ Translational Medicine Department, La \\ Sapienza University, Rome; ${ }^{2}$ Medicine and \\ Psychology Department, La Sapienza \\ University, Rome, Italy; ${ }^{3}$ Emergency \\ Medicine Department, Taunton and \\ Somerset Hospital, London, UK; ${ }^{4}$ Internal \\ Medicine Department, SS. Giovanni e \\ Paolo Hospital, Venice; ${ }^{5}$ Department of \\ Emergency Medicine, S. Giovanni Battista \\ Hospital, Turin; ${ }^{6}$ Emergency Room Unit, \\ MG Vannini Hospital, Rome; ${ }^{7}$ Association \\ of Cardiologists and Outpatients \\ Specialists (ACSA), Rome; ${ }^{8}$ Human \\ Resources Department, HRO/MDP \\ Occupational Medicine and Emergency \\ Department, Fiumicino-Ciampino, ADR \\ Rome Airport, Rome; ' Department of \\ Medical-Surgery Sciences and \\ Translational Medicine, Sant'Andrea \\ Hospital, Rome, Italy
}

\section{Abstract}

Syncope is a transient loss of consciousness due to temporary global cerebral hypoperfusion characterized by rapid onset, short duration, and spontaneous complete recovery. Syncope represents 1-2\% of emergency department (ED) visits and is coupled with a high risk for mortality, prolonged hospital admission, and immediate false diagnosis. Many patients who present to the ED with aspecific symptoms are mainly hospitalized because of diagnostic uncertainty. It is always very important to immediately distinguish syncope of cardiac and non-cardiac origins. Cardiac syncope has higher risk for mortality especially for sudden cardiac death, while non-cardiac one shows risk of repeated events of syncope with poor quality of life. Sudden cardiac death is defined as rapid and unexpected natural death due to cardiac etiology. Researchers from the GREAT Network hypothesized to evaluate some novel biomarkers in order to test acute cardiac condition that can suggest the presence of heart structural diseases, heart failure, and electrical disorders. The primary objective of this study is to test the diagnostic performance from patient history, clinical judgment, and novel biomarkers in the diagnosis of cardiac syncope in patients admitted to the ED. The trial is designed as a prospective international multicenter observational study accounting for 730 patients aged over 40 admitted to the ED with syncope within the last $12 \mathrm{~h}$.

A multimarker approach combining markers of different origin and mode of relapse, should add diagnostic information to correctly identify the cardiac conditions and to therefore be pertinent in the early diagnosis of cardiac syncope and in the prediction of cardiac events including sudden death. Future data should be needed to confirm the hypothesis presented here.

\section{Syncope}

Syncope is a temporary loss of consciousness (T-LOC) due to transitory global cerebral hypoperfusion. It is characterized by rapid onset, short duration, and spontaneous complete recovery. ${ }^{1}$

The origin of syncope is very important and within many causes we can group the causes in: cardiac and not cardiac origins).$^{1,2}$ Cardiac syncope, due to electrical disorders and structural heart diseases, ${ }^{1,3}$ has a higher risk of mortality especially for sudden death. Syncope is very frequent in the emergency departments (ED) ${ }^{1}$ and it represents $1-2 \%$ of ED visits. ${ }^{4}$ Considering the wide variety of possible differential diagnosis and prognosis, and the fact that the reported falls are often not documented, many patients who arrive at the ED with such symptoms are hospitalized.

The following epidemiological data come out from literature: the incidence of syncope in the ED varies between 1-1.5 and 13-83\% of the admitted patients. ${ }^{5}$ We know that $30 \%$ of the adult population will have at least one episode of syncope during their life and $30 \%$ of them are hospitalized since no clear causes are found. In the ED the annual cost for syncoperelated hospitalization is estimated to be 2.4 billion, with a mean cost of $\$ 5400$ per hospitalization. ${ }^{6}$ The ED's evaluation of patients with syncope is problematic because patients are often asymptomatic. It is very important to identify patients with cardiac syncope early because they could have a potentially more serious outcome than patients with non-cardiac syncope.

The use of laboratory biomarkers has been proposed for the stratification of the risks in patients with syncope. Troponin is been proposed as very reliable in syncope differential diagnosis and prognosis. ${ }^{7} \mathrm{~N}$-terminal-pro brain natriuretic peptide (NT-proBNP) and brain natriuretic peptide (BNP) are potential biomarkers proposed in differential diagnosis of
Correspondence: Salvatore Di Somma, Department of Medical-Surgery Sciences and Translational Medicine, Sant'Andrea Hospital, via di Grottarossa 1035-1039, 001891 Rome, Italy. Tel. +39.06.3377.5581 - Fax: +39.06.3377.5890. E-mail: salvatore.disomma@uniroma1.it

Key words: syncope, sudden cardiac death, biomarker.

Contributions: the authors contributed equally.

Conflict of interests: the authors declare no potential conflict of interests.

Received for publication: 15 February 2013.

Revision received: 27 August 2013.

Accepted for publication: 27 August 2013.

This work is licensed under a Creative Commons Attribution 3.0 License (by-nc 3.0).

(C) Copyright R. Marino et al., 2013

Licensee PAGEPress, Italy

Emergency Care Journal 2013; 9:e21

doi:10.4081/ecj.2013.e21

syncope. It has been showed that NT-proBNP increases in patients with cardiac syncope compared to patients with non-cardiac syncope. ${ }^{8}$ Some data showed that BNP is a good marker in risk stratification of cardiac syncope in ED. ${ }^{9-11}$

An accurate diagnosis for cardiac causes of syncope is essential to reduce the risks of sudden cardiac death or the re-occurrence of syncope. ${ }^{12}$ Emergency physicians need new helpful strategies in finding the correct origin of syncope. For this reason researchers hypothesized some novel biomarkers to test acute cardiac condition and able to distinguish: heart structural diseases [as myocardial ischemia tested by high sensitive troponin (hs-cTn)], heart failure [tested by mid regional (MR) natriuretic peptide type A (Pro ANP) and Proadrenomedullin (ProADM) ], and electrical disorders [as cardiac arrhythmias test Pro ANP and Proendothelin type 1(Pro-ET1)]. Structural diseases and electrical disorders can be the causes of cardiac syncope. It has been showed in the literature that high sensitive troponin increases in acute myocardial infarction and it is a good marker of diagnosis. ${ }^{13}$ Recently high sensitive troponin has been hypothesized to be a good marker in patients with syncope. ${ }^{14}$ Several studies have demonstrated that MR Pro ANP increases in patients with cardiac arrythmias and in patients with heart failure. ${ }^{15,16}$ It has been shown that Pro ADM, a potent vasodilatator, increases alone and together with NT pro BNP, in acute myocardial infarction. ${ }^{17,18}$ It has also been showed that copeptin is a better marker than NT pro BNP ${ }^{18,19}$ in diagnosis and even in 
prognosis of myocardial infarction. Proendothelin 1 (Pro-ET1), a 21 amino acid peptide, is a potent vasoconstrictor isolated from vascular endothelial cells; it exerts its vascular effects by activating the receptors for proendothelin type A and B on smooth cells causing increasing cellular calcium. ${ }^{20} \mathrm{~A}$ previous report shows how Pro-ET1 increases during acute myocardial infarction; the increasing of the marker is proportional to the severity of the disease. ${ }^{17,21}$ It was recently demonstrated that Pro-ET1 is linked to malignant arrhythmias in patients with chronic heart failure. ${ }^{22}$ On the basis of its mechanism of action, ProET1 has been proposed in a patented study as a marker in predicting risk of tachyarrythmic events. Plasma level of Pro-ET1 greater than 73 $\mathrm{pg} / \mathrm{L}$ expressed in median, identifies a higher risk of tachyarrhythmia events than plasma level lower than $73 \mathrm{pg} / \mathrm{L}$. The prognostic value of Pro-ET1 has been compared to BNP. Also, Pro-ET1 is a better predictor of tachyarrhythmia events compared to BNP, and a ProET1 cutoff of $75 \mathrm{pm} / \mathrm{L}$ predicts tachyarrhythmia events.22

On the basis of previous research, we proposed the Basel Syncope Evaluation Study. The primary objective is to test the diagnostic performance of patient history, clinical judgment, and novel biomarkers in the diagnosis of cardiac syncope in patients admitted to the ED. The trial is designed as a prospective international multicenter observational cohort study coordinated by the University Hospital Basel, Switzerland. Inclusion criteria were patients aged over 40 admitted to the ED with syncope within the last $12 \mathrm{~h}$. Exclusion criteria are: patients without loss of consciousness, certain neurological causes (recurrent epilepsy), hemiplegia on arrival, aged $<40$ years. All patients underwent patient history, physical examination, standard blood tests, a 12-lead electrocardiogram (ECG), and clinical judgment [a visual analog scale (VAS)]. Blood samples were also taken, frozen and analyzed for the following markers: hs-cTn, BNP, NTproBNP, MR-proANP, Copeptin, pro-ADM, proendothelin-1. The study includes a telephonic follow up at 6-12-24 months. The enrollment of Sant'Andrea Hospital (Rome, Italy), from July to October 2012 included 14 patients. Four patients had a clinical suspect of cardiac syncope, the mean of clinical judgment was $79.83 \%$, and they were all hospitalized. The other 10 patients had a clinical suspect of noncardiac syncope, the mean of clinical judgment was of $26.6 \%$, and 5 out 10 patients were hospitalized. The study data will be analyzed at the end of enrollment in 2013 at reaching of 730 patients enrolled. Syncope has many important aspects. It has a high risk of mortality, prolonged hospital admission and immediate diagnosis missing.

\section{Sudden death}

Sudden cardiac death is defined as sudden and unexpected natural death due to cardiac etiology that occurs instantly and apparently in the absence of symptoms, or within one hour from the onset of acute symptoms, or a rapid deterioration of the clinical conditions in individuals with no known potentially fatal diseases, or in individuals with pre-existing chronic heart disease, in which death occurs suddenly and unexpectedly with respect to both time and way. The evaluation of epidemiological data about sudden cardiac death is difficult because of the different incidence among populations with low or high cardiovascular risk, and among different geographical areas. In addition, we have to consider that statistical data are not unique. There are two peaks of maximum incidence: between 0 and 6 months and between 45 and 75 years. Sudden death occurs more frequently in men than in women: the average ratio is $3: 1$ (7:1 between 55 and 65 years and 2:1 between 64 and 75 years). Sudden death is more frequent due to ventricular fibrillation (75-80\%). In the Framingham Heart Study, the 5-year cumulative incidence of sudden cardiac death was approximately $7 \%{ }^{23}$ Ventricular tachyarrhythmias are fatal events that usually occur even during the chronic phase of the disease. The prevention of sudden cardiac death is largely a problem of primary care because less than $5 \%$ of people who experience sudden cardiac death have a history of previous episodes of ventricular tachycardia or ventricular fibrillation with syncope. So sudden death can be avoided if we detected early patients with syncope due to cardiac origins for example arrhythmias.

\section{Conclusions}

When evaluating patients with syncope, ED physicians must promptly distinguish those with high or low risk for acute events and admit only them at high risk, while patients with medium risk should be admitted to short observation unit. We can hypothesize that in patients with syncope some novel biomarkers might be clinically useful in quickly identify patients at high risk of death. A multimarkers approach, combining markers of different origin and mode of relapse, should add diagnostic information to correctly identify the cardiac conditions and therefore to be pertinent in the early diagnosis of cardiac syncope and in the prediction of cardiac events.

\section{References}

1. Moya A, Sutton R, Ammirati F, et al. Guidelines for the diagnosis and management of syncope (version 2009). Eur Heart J 2009;30:2631-71.

2. Brignole M, Alboni P, Benditt DG, et al. Guidelines on management (diagnosis and treatment) ofsyncope-update 2004 . Executive summary. Eur Heart J 2004;25: 2054-72.

3. Soteriades ES, Evans JC, Larson MG, et al. Incidence and prognosis of syncope. New Engl J Med 2002;347:878-85.

4. Baron-Esquivias G, Martínez-Alday J, Martín A, et al. Epidemiological characteristics and diagnostic approach in patients admitted to the emergency room for transient loss of consciousness: Group for Syncope Study in the Emergency Room (GESINUR) study. Europace 2010;12:86976.

5. Thiruganasmbandamoorthy V, Hess EP, Turko E, et al. Outcomes in Canadian emergency department syncope patients: are we doing a good job? J Emerg Med 2013;44:321-8.

6. Sun BC, Emond JA, Camargo Jr CA. Direct medical costs of syncope-related hospitalizations in the United States. Am J Cardiol 2005;95:668-71.

7. Reed MJ, Newby DE, Coull A, Prescott RJ, Gray AJ. Diagnostic and prognostic utlity of troponin estimation in patients presenting with syncope a prospective cohort study. Emerg Med J 2010;27:272-6.

8. Pfister R, Deidrichs H, Larbig R, Erdmann E, Schneider CA. NT-PROBNP for differential diagnosis in patients with syncope. Int J Cardiol 2009;133:51-4.

9. Reed MJ, Chir MB, Newby DE, et al. The rose (risk stratification of syncopein the emergency department) study. Clinical Researche 2010;55:713-21.

10. Christ M, Sharkova J, Bayrakcioglu S, et al. B-type natriuretic peptide levels predict event-free survival in patients with implantable cardioverter defibrillators. Eur J Heart Fail 2007;9:272-9.

11. Tanimoto K, Yukiiri K, Mizushige K, et al. Usefulness of brain natriuretic peptide as a marker for separating cardiac and noncardiac causes of syncope. Am J Cardiol 2004;93:228-30.

12. Moya A, Sutton R, Ammirati F, et al. Guidelines for the diagnosis and management of syncope (version 2009): the task force for the diagnosis and management of syncope of the European Society of Cardiology (ESC). Eur Heart J 2009;30: 2631-71.

13. Keller T, Zeller T, Ojeda F, et al. Serial changes in highly sensitive troponin I 
assay and early diagnosis of myocardial infarction. JAMA-J Am Med Assoc 2011;306:2684-93.

14. Reed MJ, Mills NL, Weir CJ. Sentive troponin assay predicts outcome in syncope. Emerg Med J 2012;29:1001-3.

15. Rossi A, Enriquez S, Burnett JC, et al. Natriuretic peptide levels in atrial fibrillation: a prospective hormonal and Dopplerechocardiographic study. J Am Coll Cardiol 2000;35:1256-62.

16. Tuinenburg AE, Van Veldhuisen DJ, Boomsma F, et al. Comparison of plasma neurohormones in congestive heart failure patients with atrial fibrillation versus patients with sinus rhythm. Am J Cardiol 1998;81:1207-10.

17. Behnes M, Papassotiriou J, Walter T, et al.
Long-term prognostic value of mid-regional proadrenomedullin and C-terminal proendothelin-1 in patients with acutemyocardial infarction. Clin Chem Lab Med 2008;46:204-11.

18. Khan SQ, O'Brien RJ, Struck J, et al. Prognostic value of midregional proadrenomedullin in patients with acute myocardial infarction: the LAMP (Leicester Acute Myocardial Infarction Peptide) study. J Am Coll Card 2007;49: 1525-32.

19. Reichlin T, Hochholzer W, Stelzig C, et al. Incremental value of copeptin for rapid rule out of acutemyocardial infarction. J Am Coll Cardiol 2009;54:60-8.

20. Schneider MB, Boesen EI, Pollock DM. Contrasting actions of endothelin ET(A) and ET(B) receptors in cardiovascular disease. Annu Rev Pharmacol 2007;47:731-59.

21. Tomoda H. Plasma endothelin-1 in acute myocardial infarction with heart failure. Am Heart J 1993;125:667-72.

22. Struck J, Morgenthaler N, Bergmann A, Müller C. Pro-endothelin-1 levels for the prediction of risk of tachyarrhytmic events. Available from: http://www. faqs.org/patents/app/20110111525

23. Berger CJ, Murabito JM, Evans JC, et al. Prognosis after first myocardial infarction: comparison of Q-wave and non-Q-wave myocardial infarction in the Framingham Heart Study. JAMA-J Am Med Assoc 1992; 268:1545-51. 